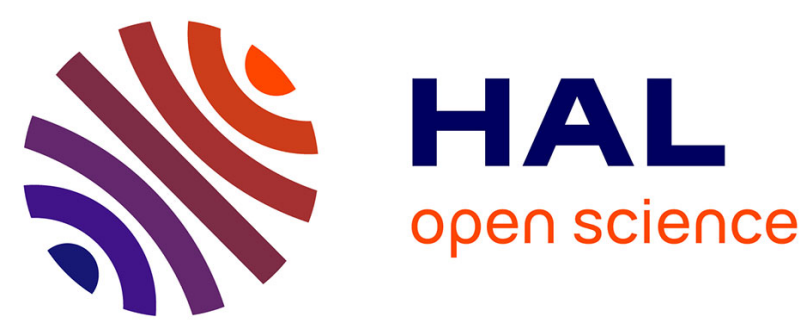

\title{
Detection and localization of a gear fault using automatic continuous monitoring of the modulation functions
}

Xavier Laval, Nadine Martin, Pascal Bellemain, Corinne Mailhes

\section{- To cite this version:}

Xavier Laval, Nadine Martin, Pascal Bellemain, Corinne Mailhes. Detection and localization of a gear fault using automatic continuous monitoring of the modulation functions. WCCM 2019 - Second World Conference on Condition Monitoring, Dec 2019, Singapour, Singapore. hal-02327201

\section{HAL Id: hal-02327201 \\ https://hal.science/hal-02327201}

Submitted on 22 Oct 2019

HAL is a multi-disciplinary open access archive for the deposit and dissemination of scientific research documents, whether they are published or not. The documents may come from teaching and research institutions in France or abroad, or from public or private research centers.
L'archive ouverte pluridisciplinaire HAL, est destinée au dépôt et à la diffusion de documents scientifiques de niveau recherche, publiés ou non, émanant des établissements d'enseignement et de recherche français ou étrangers, des laboratoires publics ou privés. 
Detection and localization of a gear fault using automatic continuous monitoring of the modulation functions

\author{
X. Laval ${ }^{\dagger}$, N.Martin ${ }^{1}$, P.Bellemain ${ }^{1}$ and C.Mailhes ${ }^{2}$
}

†. Univ. Grenoble Alpes, CNRS, Grenoble INP, GIPSA-Lab, 11 rue des Mathématiques, Grenoble Campus BP46, F-38402

SAINT MARTIN D'HERES, France

${ }^{\dagger}$ E-mail: xavier.laval@gipsa-lab.fr

${ }^{1}$ Univ. Grenoble Alpes, CNRS, Grenoble INP, GIPSA-Lab

${ }^{2}$ Univ. of Toulouse, IRIT/INPT-ENSEEIHT /TéSA.

\begin{abstract}
In the context of automatic and preventive condition monitoring of rotating machines, this paper presents a case study of a naturally-worn parallel straight gear by monitoring the evolution of the modulation functions. The Hilbert demodulation is automatically performed considering only the frequency content of the signals detected by the AStrion software. The gear has been worn over 3000 hours with a constant axial load. A particular focus is set on the amplitude modulation function in order to assess its efficiency to characterize both the severity of the wear and the most worn part of the gear. The results are confronted with on-site observation of the teeth. For this purpose, the evolution of both amplitude and phase modulations over several meshing harmonics are compared, as well as demodulation on both original and residual signals. Indicators to automatically classify the wear are discussed.
\end{abstract}

Keywords: Condition monitoring, signal processing, Hilbert demodulation, gear fault detection, continuous surveillance

\title{
1. Introduction
}

Gearboxes are very important parts of mechanical systems. An unforeseen failure in an industrial context can lead to important shutdowns and replacement costs. Thus, the need of a functional and efficient condition monitoring applied to these parts is crucial. However, the day-to-day preventive maintenance consists mainly in monitoring standard features such as peak-to-peak, root mean square or Kurtosis. However, these methods are limited when the goal is an early fault detection of low vibrations embedded in strong other vibrations. A lot of technics, based on advanced signal processing are reviewed in [1] and [2] such as wavelet [3], empirical mode decomposition [4], Teager-Kaiser energy operator [5]. A common method is the Hilbert demodulation, which efficiency has been proven by McFadden [6] and Randall [7].

This paper presents a case study of a gearbox wear through automatic methods with the AStrion software using the Hilbert demodulation method. AStrion is a preventive maintenance software, developed coinjointly between the GIPSA-lab in Grenoble, France and the IRIT in Toulouse, France. This software, which principles have been presented in [8], [9] and [10], operates by automatically 
detecting peaks and classifying them in harmonics and sideband families. The sideband families can be automatically demodulated to estimate the temporal modulation functions. This paper aims at characterizing the wear by monitoring the evolution of these functions with simple operators requiring no a priori knowledge of the system. The aim is to propose automatic methods to characterize spallings without mechanical modelling, using sensors on different positions to give credit to the diagnosis. The demodulation process will be shortly presented first. The GOTIX bench, from where the data come from, will be introduced in a second part. The monitoring of the modulation functions on a set of sensors will be presented in a third part. Specific indicators are defined from these modulation functions. The fourth part shows the ability of the indicator trends along timestamp signals to characterize the wear. Discussion of the method and its limitations in operational context will be undertaken in the last part.

\section{Demodulation process in AStrion and physical meaning of modulation functions}

From the identified harmonics and sideband families, AStrion is able to demodulate a specific family in signals where this family has been detected. The demodulation module first applies a multi-rate filter on a band corresponding either to the number of detected sidebands or a specific user-defined number of sidebands. This narrow band spectrum is then shifted to zero, before applying a synchronous averaging at the modulation frequency. Finally, the Hilbert transform is applied without removing the deterministic part. The method has been presented in details in [11]. After demodulation, 3 temporal signal functions representing the amplitude modulation (AM), the phase modulation (PM) and the frequency modulation (FM) are given over one cycle of the modulation frequency.

If the demodulated harmonic corresponds to a meshing frequency, the AM is the envelope of the gearbox vibration. Its mean corresponds to the energy of the demodulated harmonic. It can thus be described as the instantaneous acceleration, which is an image of the force generated at the point of meshing. If the modulating frequency corresponds to a shaft connected to the gearbox, once applied the synchronous averaging, the AM represents the variations of the force generated by the meshing over one rotation of the gear. This force is influenced in particular by shaft misalignments, load asymetry and teeth meshing. Furthermore, a tooth with a defect such as spalling has a different stiffness compared to a healthy one [12]. Since the acceleration measure is directly influenced by stiffness variation, it is expected that for a damaged gear, the AM variations will be representative of the fault.

The FM is the derivative of the PM and represents the instantaneous frequency, its mean being equal to the demodulated harmonic frequency. In the case of a gearbox modulated by a shaft, after the synchronous averaging at the modulation frequency, the FM will represent the instantaneous speed of meshing. It can be influenced for example by fissured teeth. However, in particular for low energy harmonics, or for strong spalling, the AM can drop close to zero and be folded, causing a $180^{\circ}$ phase 
shift and an artificial surge in the FM. Thus the FM is more problematic to use, since it can lead to false interpretation.

\section{Data from the GOTIX experimental bench}

The GOTIX bench, hosted in the GIPSA-lab, is an experimental setup consisting in a gearbox driven by an asynchronous motor and loaded by a DC-generator. A synchronized set of data comprising vibrations, currents, voltages and angular positions, are recorded through an OROS38 acquisition system. More information can be found on the GOTIX website http://www.gipsa-lab.grenobleinp.fr/projet/gotix/presentation.html from which data can be downloaded. The main experiment is a long-term natural wear experiment on a parallel straight tooth multiplier gearbox composed of a $Z_{\text {gear }}=57$ teeth gear and a $Z_{\text {pinion }}=15$ teeth pinion. Thus, the vibration signals are all dominated by the meshing harmonics. The different harmonics are modulated by both the input and output shafts. AStrion is able to detect all harmonics and modulations automatically, up to 57 between the strongest harmonics. AStrion results in a frequency zoom are presented in Figure 1. It is noticeable that the sidebands $+/-19$ of the gear shaft rotation are predominant. This is due to the assembly phase frequency, generated by the fact that the number of gear teeth and pinion teeth are not prime numbers: $\frac{Z_{g e a r}}{19}=$ $\frac{Z_{\text {pinion }}}{5}=3$. In order to avoid interferences from this frequency and its sidebands, the maximum number of sidebands has been set to 9 on each side of the harmonic.

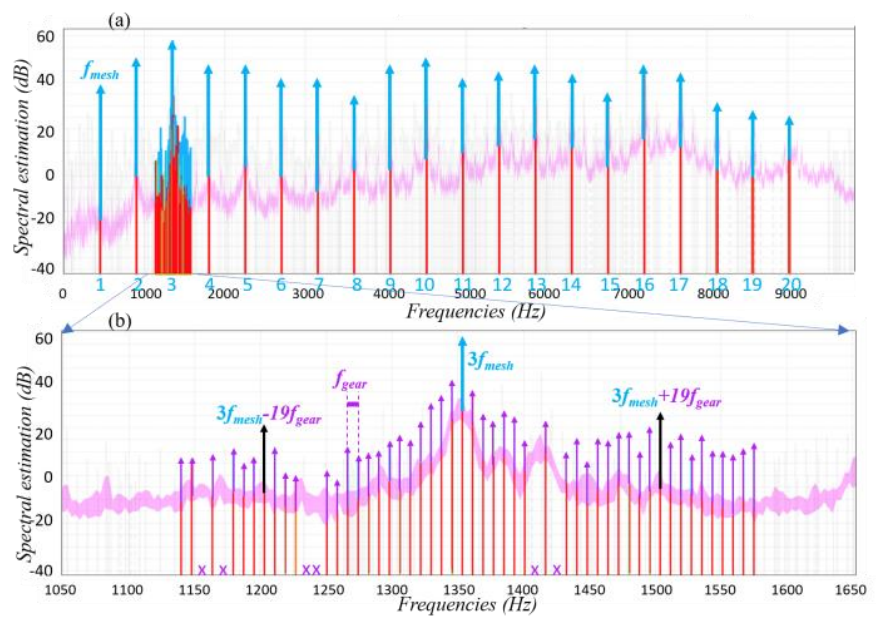

Figure 1: AStrion results in a frequency zoom of a GOTIX signal estimated. The meshing harmonics are identified in (a), and an additional zoom shows the identified sidebands around the third harmonics in (b).

The bench has been rotating for over 6000 hours with the same pair of gears, slowly wearing them in a completely natural way. Recordings were performed every 5 hours. Currently, the gears are worn, with spalling marks on several teeth, but not yet out of use. The last change in the operating conditions happened 3300 hours ago for technical resaons. Since then, the speed and load have been near constant at respectively $475 \mathrm{rpm}$ and $760 \mathrm{Nm}$ on the driving gear shaft. Thus, the study has been performed on these last 3300 hours, with 650 signals investigated, each signal corresponding to 80 seconds of 
recording. Six accelerometers are located at 3 different positions, measuring 2 axes at each position. The considered sensors in the following parts, denominated sensor 1 and sensor 2, are both radially orientated. Sensor 1 is on the gear while sensor 2 is on the pinion. AStrion results in Figure 1 show a predominance of the third harmonic. This is true for the 4 radial accelerometers on GOTIX and probably indicates a system resonance. However, the choice here is to not take into account any resonance but to demodulate all harmonics and to compare their similarities in order to identify the potential faulty parts of the gear.

\section{Monitoring of the modulation functions}

In order to obtain comparable modulation functions, the signals are all synchronized using a top-tour signal from the modulating gear shaft. Thus, resulting modulation functions start from the same angular position of the gear. Harmonics with a sufficient number of sidebands are all investigated, whatever their amplitude. Furthermore, the AM and FM functions are normalized by their mean value in order to compare only the shape of these functions and be independent from their harmonic amplitude and frequency variations. These two features are also monitored independently by AStrion. As can be seen on Figure 2, for sensor 1, the different functions present a continuous evolution as the gear is slowly wearing itself.

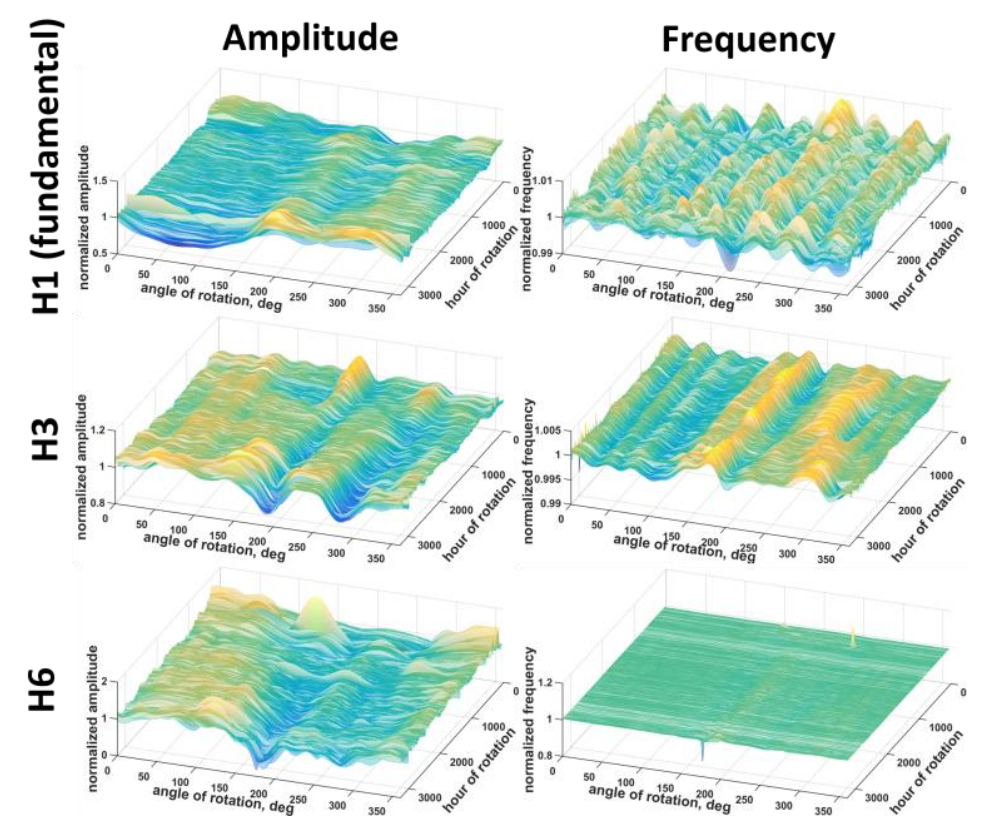

Figure 2: Evolution of AM and FM functions for 3 different harmonics (H1, H3 and H6) over the hours of gear rotation for sensor 1. Each function length corresponds to one gear rotation, from $0^{\circ}$ to $360^{\circ}$.

One can already notice trends in these plots, in particular around $200^{\circ}$ and $280^{\circ}$ : at these angular positions, AM seems to present a significant evolution, either as an increase (H1) or a decrease $(\mathrm{H} 3$ and H6). This is also true when studying other harmonics. However, it can be very long and fastidious to study each of these harmonics for every gear present in a system. The evolution is also present on FM, 
but can be masked by important peaks, visible on H6 in particular. These peaks are issued from a $180^{\circ}$ phase shift in the PM, caused by the folding of the AM when its value comes close to zero. This effect is illustrated in Figure 3. They can be problematic in the context of automatic detection. They can however be used to automatically remove AM presenting this folding, their shape being distorted.
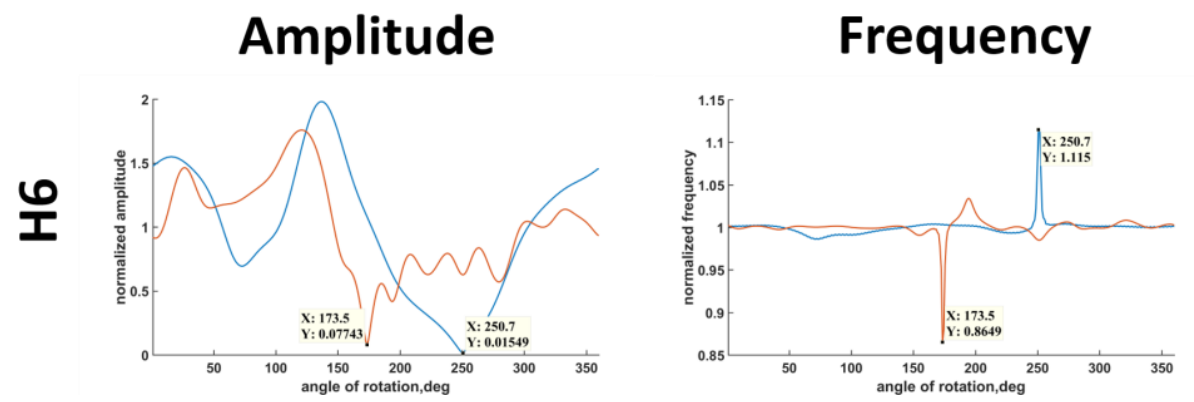

Figure 3: AM and FM from the 6th harmonic for 2 folded cases. The link between the FM peaks and the AM folding is clearly visible on datatips

In order to confirm the diagnostic, it can be interesting to compare accelerometers at different positions. Figure 4 shows AM for 2 different accelerometers, among the 8 available in the system, both oriented radially: accelerometer 1 is located on the pinion shaft while accelerometer 2 is located on the gear shaft. While differences are visible, the trends are similar. By automatically demodulating both, it is possible to confirm or refute a diagnostic.

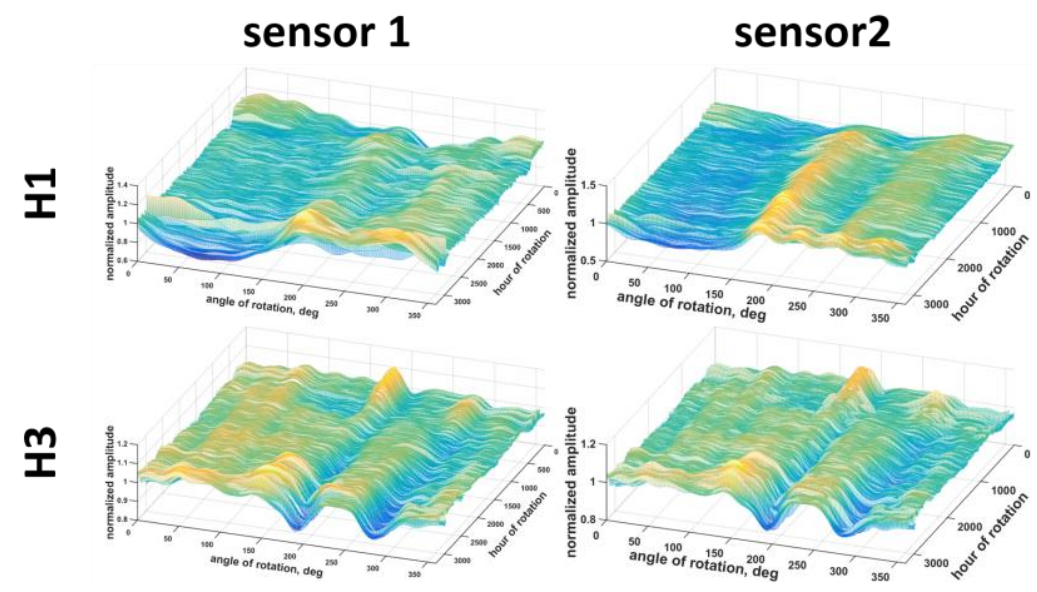

Figure 4: AM for $\mathrm{H} 1$ and $\mathrm{H} 3$ for sensors 1 and 2 from 2 different accelerometers

Even if a confirmed operator could establish a diagnosis, the process of scanning the demodulation functions for every harmonic generated by every mechanical elements of a complex system can be very time-consuming and inefficient. Hence the necessity to have features representing the evolutions that are monitorable with automatic classifications technics.

\section{Demodulation function features}

The aim of this part is to extract as much information as possible from the AM and FM functions using simple features. Thus, only classical features such as peak-to-peak (PP), root mean square (RMS) and 
Kurtosis $(\mathrm{K})$ values have been used. First, these features are computed for each measure on the global AM functions. But in order to improve the method as well as the number of features available for the automatic classification, a gliding feature is computed on each function with a rectangular angular window of length 38 degrees which amonts to dividing the functions in 19 parts, with a $50 \%$ overlap between each part to avoid side effects. As shown in [11], the filtering due to the choice of the demodulation band, +/- 9 sidebands in the present case, strongly alters the shape of the modulation functions. The choice of 19 parts is empirical, and corresponds to $N_{\text {parts }}=n_{\text {sideband left }}+$ $n_{\text {sideband right }}+1$. It can thus be determined automatically. When linking it to the mechanics, each part contains 3 teeth, plus an overlapping of around 1 tooth and a half on each side. The first six harmonics are examined. In the following, the x-axis is labelled with zone numbers, each zone cooresponding to 3 teeth of the gear as above-mentioned. Figure 5 shows the PP feature across the 19 angular zones over the 3300 hours of rotation. The AM functions of H1, H3 and H6 highlight angular zones where PP increases, in particular in zones 9 and 10, reflecting the slow wearing of the gear.
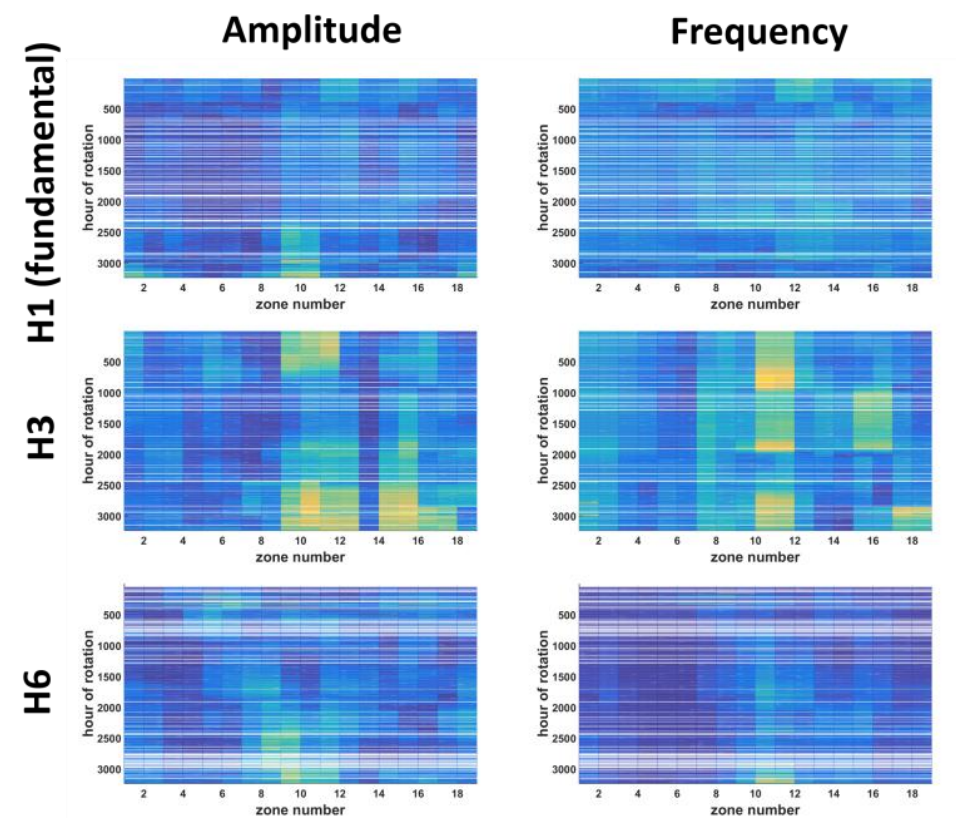

Figure 5: Gliding Peak-to-peak features for 3 harmonics on amplitude and frequency modulations functions and for all the time-stamps. The blank lines correspond to the signals for which the modulation sidebands have not been detected. The $\mathrm{x}$-axis corresponds to 19 angular zones of 3 teeth each.

However, this phenomenon is not so clear on the FM evolution. As seen in Figure 5, H1 FM does not present any evolution, while $\mathrm{H} 3$ shows some maximum in zones 10 and 11, but it does not evolve continuously. Only H6 shows an evolution. Focusing on the AM, Figure 6 confirms the interest of the PP feature, both on the whole AM and on different zones. For clarity and manual comparison purpose, the features have been normalized (divided by their mean value) for every figure. In an automatic context, this step may not be necessary. However, a median filter has also been applied to smooth the results. Since its purpose is also to improve the classification results, this step is more important. 

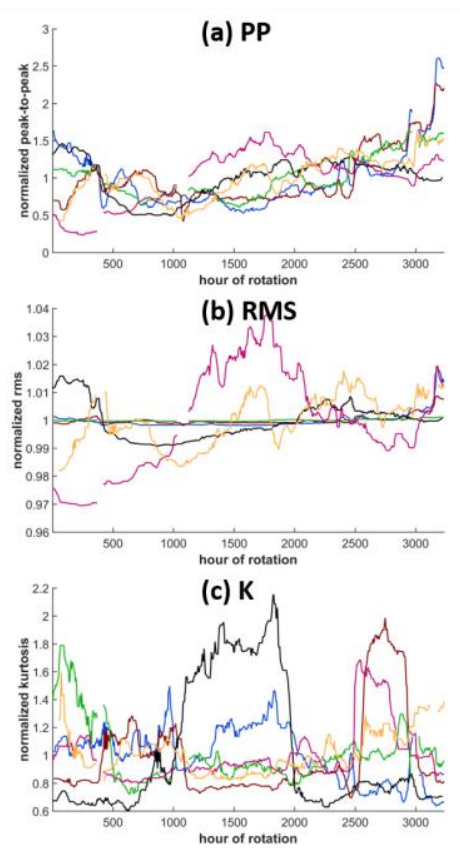
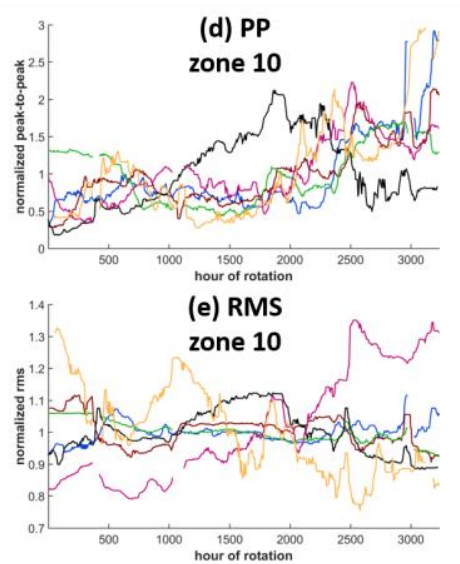

(f) $\mathrm{K}$

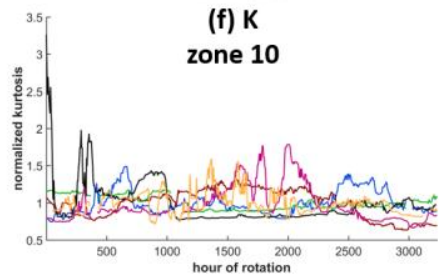

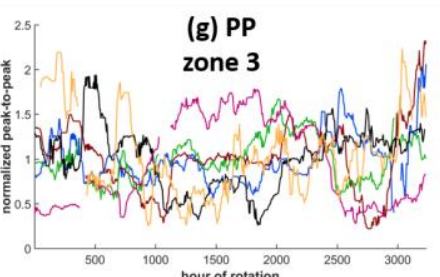

(h) PP

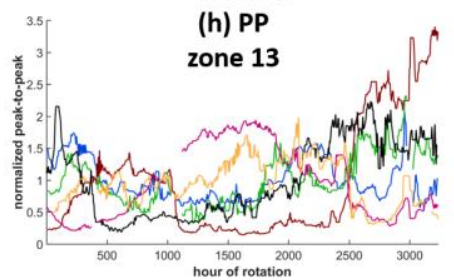

(i) PP

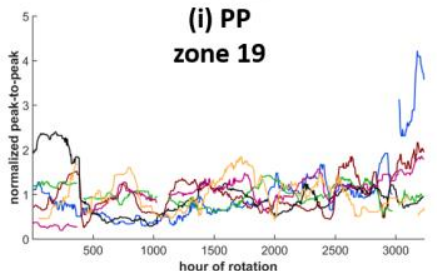

Figure 6: For harmonics H1 to H6, PP, RMS and Kurtosis on the whole amplitude modulation function (a), (b), (c). PP, RMS and Kurtosis for zone 10 (d), (e), (f). PP for zones 3, 13 and 19 (g), (h), (i)

The PP value on the whole AM (a) steadily increases for 4 harmonics. Furthermore, it increases for 5 harmonics in zone 10 (d), all of them starting their increase around the same moment, between 2000 and 2400 hours of rotation. Only H4 in fact starts to decrease at this moment, after a steadily increase. This shows that this zone in particular is more worn than the rest of the gear. On the other zones, only zone 13 (h) is showing signs of wear with 3 on 6 harmonics increasing. On the contrary, no trend can be discerned on zones 3 (g) and 19 (i). The results from Kurtosis and RMS, whether on the whole AM (b and c) or in the worn zone 10 (e and f) are also inconclusive. The fact that the PP increases in a particular zone for several harmonics is a strong validation of the PP increasing on the whole AM. It increases the likelihood of an advanced wear on this zone, and can reinforce an automatic diagnostic. When inspecting the state of the gear teeth in Figure 7, it is confirmed that the zone 10 is the most worn. Indeed, large spalling marks on teeth 30,31 and 46 can be seen, the largest being on teeth 31 . Teeth 30 and 31 belongs to zone 10. Smaller spalling marks have been observed on 18 teeth, such as tooth 21 .

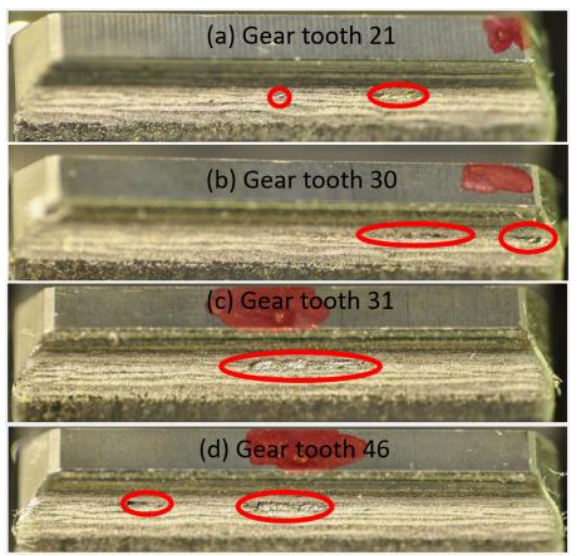

Figure 7: teeth 21, 30, 31 and 46 from the gear of the GOTIX bench. Circled in red are spalling marks 


\section{Limitations of the method}

The strongest limitation of the presented method is probably the fact that this method needs to have quite stable operating conditions, in particular speed and load, for each measure. The shapes of modulation functions are strongly dependent on the excitation of the system. This article shows that with constant operating conditions, such as on an experimental bench like GOTIX, the AM and FM present a continuous evolution, pertinent for the detection of defects. However, in the context of industrial exploitation, it is rarely the case. A solution could be to monitor the state parameters (speed and torque in particular) to trigger a measurement only when the system is operating at a predefinite condition. It can work for systems remotely and continuously monitored, such as wind turbines. When the measures are performed by technicians, they can previously set these conditions before performing the measurements.

Another limitation is the necessity to have angularly synchronized measures, starting all from the same gear angle. On the results presented here, the measures have all been synchronized using a top-tour on the gear shaft. However, these are not always present and can not be mounted easily in industrial context.

The last limitation is the changes in the system due to maintenance: a greasing for example is susceptible to change the profile of the AM function brutally and needs to be taken into account in the context of automatic monitoring, by a feed of information to the monitoring software

\section{Conclusions and perspectives}

This paper presented a case study of a gearbox natural wearing and how to automatically assess it by monitoring the AM and FM function evolution for several harmonics. It has been shown that the wear is highlighted in the very continuous evolution of the AM, more than the FM, and that a clear indicator of it is the use of the peak-to-peak feature, both on the whole AM and on specific parts associated to the wornest part of the gear. The confirmation that the wear is concentrated on a specific part of the gear, as well as the fact that other sensors presents the same characteristics, is used as a double check to ensure the fiability. The double advantage of dividing the AM from several harmonics in several zones is both to provide more features for an automatic classification, increasing the fiability of the alarm raised, and to provide technicians a zone to check on the gear and decide if the gear should be replaced.

The GOTIX experiments on the worn gear will resume after a reparation of the driving system, and will go on until the gearbox breaks naturally. It will be interesting then to check if the zones identified as worn now will be the one to break, and to see the future evolution of the AM functions. Furthermore, 
the same study should be conducted on other type of mechanical pieces, bearings or planetary gearbox for example, as well as in industrial context, in order to confirm its interest.

\section{References}

[1] P.Fanning, and E.P.Carden, "Vibration Based Condition Monitoring:A Review", SHM, Sage publications, Vol 3(4): pp.355-377, 2004

[2] F.P.G.Márquez et al, "Condition monitoring of wind turbines: Techniques and methods", Renewable Energy, Vol.46, pp. 169-178, Oct. 2012, https://doi.org/10.1016/j.renene.2012.03.003

[3] W.J.Wang and P.D.McFadden, "Application of wavelets to gearbox vibration signals for fault detection”, JSV, vol.192(5), pp.927-939, sep. 1995

[4] M.Liang et al, "Fault diagnosis for wind turbine planetary gearboxes via demodulation analysis based on ensemble empirical mode decomposition and energy separation", Renewable Energy vol.47 pp. 112-126, may 2012, doi:10.1016/j.renene.2012.04.019

[5] I. Antoniadou et al, "A time-frequency analysis approach for condition monitoring of a wind turbine gearbox under varying load conditions", MSSP, vol.64-65, pp.188-216, apr. 2015, http://dx.doi.org/10.1016/j.ymssp.2015.03.003

[6] P.D. McFadden, J.D. Smith, “A Signal Processing Technique for Detecting Local Defects in a Gear from the Signal Average of the Vibration", Proceedings of the Institution of Mechanical Engineers, Part C, vol.199 (43), pp. 287-292, oct. 1985. https://dx.doi.org/10.1243/PIME_PROC_1985_199_125_02

[7] R. B. Randall, "A new method of modelling gear faults". ASME, J. Mech. Des., vol.104, pp. 259-267., apr.1982 https://dx.doi.org/10.1115/1.3256334

[8] M. Firla et al, "Automatic Characteristic Frequency Association and All-Sidebands Demodulation for Detection of a Bearing Fault of a Wind Turbine Test Rig", MSSP, vol. 80, pp. 335-348, dec. 2016. https://doi.org/10.1016/j.ymssp.2016.04.036

[9] N. Martin, C. Mailhes, "Automatic Data-Driven Spectral Analysis Based on a Multi-Estimator Approach", Signal processing, vol.146, pp. 112-125, may 2018, https://doi.org/10.1016/j.sigpro.2017.12.024

[10] T. Gerber, N. Martin, C. Mailhes, "Time-frequency Tracking of Spectral Structures Estimated by a Data-driven Method", IEEE Trans. on Industrial Electronics, Special Session, vol.52 (10), pp. 6616-6626, july 2015. https://dx.doi.org/10.1109/TIE.2015.2458781

[11] X. Laval et al, "Vibration response demodulation, shock model and time tracking", CM 2018 and MFPT 2018. Nottingham, UK, 10-12 September 2018.

[12] F. Chaari et al, "Effect of spalling or tooth breakage on gearmesh stiffness and dynamic response of a one-stage spur gear transmission", European Journal of Mechanics A/Solids vol.27, pp. 691-705, july 2008, https://doi.org/10.1016/j.euromechsol.2007.11.005 